\title{
Géolinguistique
}

15 | 2015

La géographie linguistique au Brésil

\section{Atlas Lingüístico do Estado do Ceará (ALECE)}

Atlas Lingüístico do Estado do Ceará (ALECE)

Maria do Socorro Silva de Aragão

\section{(2) OpenEdition}

Journals

Édition électronique

URL : http://journals.openedition.org/geolinguistique/642

DOI : $10.4000 /$ geolinguistique. 642

ISSN : 2650-8176

\section{Éditeur}

UGA Éditions/Université Grenoble Alpes

\section{Édition imprimée}

Date de publication : 1 décembre 2015

Pagination : 137-148

ISBN : 978-2-84310-317-9

ISSN : 0761-9081

\section{Référence électronique}

Maria do Socorro Silva de Aragão, "Atlas Lingüístico do Estado do Ceará (ALECE) », Géolinguistique [En ligne], 15 | 2015, mis en ligne le 15 février 2019, consulté le 31 octobre 2020. URL : http://

journals.openedition.org/geolinguistique/642; DOI : https://doi.org/10.4000/geolinguistique.642 


\title{
Atlas Lingüístico do Ceará
}

\author{
Maria do Socorro Silva de Aragão \\ Universidade Federal da Paraíba, \\ Universidade Federal do Ceará (Brésil)
}

\section{Résumé}

L'Atlas Lingüístico do Estado do Ceará (ALECE), publié en 2010, est le résultat du travail d'un groupe de professeurs du Département de lettres vernaculaires de l'Université fédérale du Ceará, coordonné par le professeur José Rogério Fontenele Bessa et avec l'aide de professeurs invités et de consultants. Pour la sélection du réseau de points de l'ALECE ont été choisi 70 localités des microrégions homogènes, avec des caractéristiques physiques, sociales et économiques identiques. Pour la constitution du corpus nous avons interrogé 268 locuteurs, un homme et une femme dans chaque localité appartenant à des tranches d'âge s'échelonnant de 30 à 60 ans, alphabètes ou ayant un niveau de scolarité du premier degré complet. Les enquêtes sur le terrain ont été réalisées avec un questionnaire comportant 306 questions relatives à 583 concepts, réunies en 16 champs sémantiques, permettant l'analyse du phonétisme et du lexique du parler du Ceará. L'ALECE comporte deux volumes. Le volume 1 contient une introduction avec les aspects historiques, la présentation du projet, l'orientation théorique et la méthodologie utilisée. Le volume 2 présente des indications pour la lecture de l'atlas, les cartes phonétiques et lexicales avec une légende indiquant les conventions et les symboles utilisés, un glossaire et des références bibliographiques. Les entrées, avec la transcription phonétique, indiquent la réalisation, la classe et la catégorie grammaticales, le concept, le numéro de la question, les caractéristiques de l'informant et de la localité. L'Atlas Lingüístico do Ceará trouve toute sa place parmi les autres atlas consacrés à la région Nord-Est, à savoir l'Atlas Prévio dos Falares Baianos (1963), l'Atlas Lingüístico da Paraíba (1984), l'Atlas Lingüístico de Sergipe (1987), l'Atlas Geo-sociolínguístico do Litoral Potiguar (1990), l'Atlas Lingüístico de Sergipe II (2005), l'Atlas 
Lingüístico do Iguatu - Ceará (2009), l'Atlas Lingüístico da Mata Sul de Pernambuco (2009), l'Atlas Lingüístico Léxico-Semântico de Capistrano - Ceará (2011), l'Atlas Lingüístico do Oeste Potiguar (2012) et l'Atlas Lingüístico de Pernambuco (2013).

\title{
Mots-clés
}

Atlas linguistiques régionaux, atlas de Ceará.

\begin{abstract}
The Atlas Lingüístico do Estado do Ceará (ALECE), published in 2010, is the work result from a group of teachers from the Departamento de Letras Vernáculas of the Federal University of Ceará, coordinated by Professor José Rogério Fontenele Bessa, with the help by visiting professors and consultants. In order to organize the network of points of the ALECE, 70 locations of the homogeneous micro regions were selected, with alike physical, social and economic characteristics. To constitute the corpus 268 informants, one man and one woman from each location were interviewed, with ages from 30 to 60 , with instruction level from illiterate to high school level. The field research had the application of a questionnaire with 306 questions, with a universe of 583 items, in 16 semantical fields, about phonetical and lexical aspects from the speech of Ceará. The ALECE was published in two volumes. The Volume 1 contains an introduction with the proceding, theoretical orientation, the project and methodology used. The Volume 2 contains orientations to read the atlas, lexical and phonetical maps, a glossary and a bibliography. All the maps have notes of the conventions used, different types of symbols. The entries come with the phonetical transcriptions of its realization, the class and grammatical category, the concept, indication of the question number and characterization of the informant and of the location. The Atlas Linguístico do Estado do Ceará is an addition to the other atlases from the Northeast Region of Brazil, such as the Atlas Prévio dos Falares Baianos (1963), Atlas Lingüístico da Paraíba (1984), Atlas Lingüístico de Sergipe (1987), Atlas Geo-sociolingüístico do Litoral Poiguar (1990), Atlas Lingüístico de Sergipe II (2005), Atlas Lingüístico do Iguatu - Ceará (2009), Atlas Lingüís-tico da Mata do Sul de Parnambuco (2009), Atlas Lingüístico Léxico-Semântico de Capistrano -Ceará (2011), Atlas Lingüístico do Oeste Potiguar (2012) and Atlas Lingüístico de Pernambuco (2013).
\end{abstract}

\section{Keywords}

Regional linguistic atlases, Ceará atlases. 
L'État du Ceará a une tradition d'études dialectales remontant au XIX siècle, avec João Capistrano Honório de Abreu qui a publié dans La Quinzaine un travail sur As Origens da Palavra Ceará (1887) et aussi William Studart auteur de A Jangada (1887), paru dans la même revue, de nombreux écrivains comme Martinz de Aguiar, Florival Seraine, Leonardo Mota, Raimundo Girão, Tomé Cabral et José Rebouças Macambira, entre autres, originaires du Nord-Est, ont écrit sur le parler du Ceará. Héritière de ces expériences, en 1978, une équipe de chercheurs de l'Universidade Federal do Ceará, issue du Centre de Humanidades, a commencé à travailler à l'élaboration de l'Atlas Lingüístico do Ceará qui a été publié en 2010.

\section{Les recherches pour l'Atlas linguistique du Ceará}

Les recherches pour la réalisation de l'Atlas Lingüístico do Ceará ont été développées en adoptant les approches de la géolinguistique moderne, en tenant compte non seulement de la variation diatopique, mais aussi diastratique, prenant en compte des locuteurs appartenant à deux niveaux d'instruction différents.

\subsection{Choix des localités}

Initialement, 70 localités ont été choisies couvrant l'ensemble de l'État: par la suite, trois d'entre elles ont été éliminées, réduisant le réseau à 67 points ${ }^{1}$.

\subsection{Choix des informateurs}

Pour chaque localité deux hommes et deux femmes ont été choisis, sur la base de plusieurs critères : être nés dans la ville enquêtée; appartenir à une tranche d'âge située entre 30 et 60 ans; avoir une dentition en bon état; avoir un niveau d'instruction allant de l'analphabétisme à un niveau d'école

1. Les communes sélectionnées sont : Tianguá, Viçosa do Ceará, São Benedito, Ubajara, Ibiapina, Guaraciaba du Nord, Carnaubal, Pacoti, Guaramiranga, Mulungu, Baturité, Aracoiaba, Capistrano, Itapiúna, Aratuba, Rédenção, Sobral, Merioca, Uruoca, Coreaú, Massapé, Canindé, Beberibe, Uruburetama, Brejo Santo, Jardim, Missão Velha, Milagres, Crato, Barbalha, Caririaçu, Farias Brito, Nova Olinda, Juazeiro, Santana do Cariri, Ipu, Nova Russas, Crateús, Tauá, Parambu, Aiuaba, Quixadá, Quixeramobim, Senador Pompeu, Mombaça, Morada Nova, São João do Jaguaribe, Limoeiro do Norte, Russas, Tabuleiro do Norte, Jaguaribe, Pereiro, Campos Sales, Assaré, Araripe, Santa Quitéria, Monsenhor Tabosa, Boa Viagem, Independência, Camocim, Acaraú, Granja, Bela Cruz, Acopiara, Iguatu, Icó et Cedro. 
primaire complet, voire à un niveau d'études secondaires (complet ou partiel); ne pas avoir quitté l'école ni la région; exercer des professions variées. Au total 261 informateurs ont été interrogés. Le fait d'avoir enregistré, systématiquement, des informateurs appartenant à deux niveaux d'instruction, à savoir analphabètes/scolarisés, permet de considérer l'ALECE comme un atlas bidimensionnel prenant en compte la variation diatopique et diastratique. Par ce choix, auquel il faudrait ajouter le fait d'avoir recours à des informateurs multiples dans chaque localité, il se différencie de la plupart des atlas de première génération, monodimensionnels, avec lesquels il partage par ailleurs l'essentiel de la démarche méthodologique.

\subsection{Le questionnaire}

Le questionnaire ALECE a été publié en 1982 par les Presses universitaires de l'Université fédérale du Ceará. Les entrées sont rassemblées en 16 champs sémantiques : la nature, le temps, l'homme, la parenté, les parties et les fonctions du corps humain, les maladies, les caractéristiques physiques de l'homme, les parties du corps, fonctions, les jeux, les objets d'usage personnel, les activités et les ustensiles domestiques, la nourriture, la religion, les animaux, autres concepts, pour un total de 306 questions pour 583 items. Au début des travaux le questionnaire a été utilisé expérimentalement dans 22 localités.

\subsection{Les transcriptions graphématiques}

Avant les transcriptions phonétiques, ont été réalisées les transcriptions graphématiques des éléments lexicaux des cartes et du glossaire, dans l'ordre alphabétique de la langue portugaise, en restant le plus proche possible, de la façon de parler des enquêtés, comme dans les mots : uruvalho, cicrone, librina, diluve, relampo.

\subsection{Les transcriptions phonétiques}

Les transcriptions phonétiques des interviews ont été réalisées par des assistants de recherche formés à cet effet et contrôlées par les enseignants de phonétique. Le système adopté comprend des symboles de l'Alphabet phonétique international (API), de l'Institut linguistique de Verão (ILV) dont le siège est à Brasilia, mais aussi un petit nombre de symboles et de diacritiques créés par l'équipe de l'ALECE. La transcription, large et générale, est basée sur l'impression auditive, sans l'utilisation d'une approche instrumentale. 


\section{Présentation de l'Atlas linguistique du Cearà}

L'Atlas linguistique du Ceará comporte deux volumes : le premier contient une introduction, un historique, la présentation du projet, l'orientation théorique et la méthodologie utilisée, une analyse des atlas brésiliens déjà publiés, une bibliographie dialectale du Cearà. Suit un chapitre consacré à l'atlas lui-même avec, en particulier, la présentation détaillée de l'État et de ses régions, de toutes les localités du réseau, et de tous les informateurs. Une petite section du volume présente l'inventaire des phonèmes et de leurs variantes libres et combinatoires relevées à partir des données des enquêtes publiées dans les cartes du deuxième volume. Concernant la terminologie de l'atlas, l'équipe de travail a décidé d'adopter quelques désignations particulières dans le domaine phonético-phonologique : c'est le cas, par exemple, des termes vocoïdes, semivocoïdes, contoïdes et nasalisés employés à la place de voyelles, semivoyelles, consonnes et nasales. Tous les phonèmes de la langue portugaise, comme le montrent les tableaux ci-dessous, sont présents dans le parler du Ceará, bien que leurs réalisations et leurs distributions révèlent des traits de ce parler régional :

\subsection{Contoïdes oraux et nasalisés}

\begin{tabular}{|c|c|}
\hline$/ \mathrm{p} /-[\mathrm{p}][$ 'pata] pata & $/ \mathrm{b} /-[\mathrm{b}][$ 'bata] bata \\
\hline$/ \mathrm{t} /$ - [t] [tẽpe $\int^{\prime}$ ta dzi $]$ tempestade & $/ \mathrm{d} /-[\mathrm{d}]\left[\mathrm{i}^{\prime}\right.$ dozu] idoso \\
\hline$/ \mathrm{k} /$ - [k] ['porka] porca & $/ \mathrm{g} /$ - [g] [' gŗosa] grossa \\
\hline /f/ - [f] ['fasis] faces & $/ \mathrm{v} /$ - [v] [vẽtã' nia] ventania \\
\hline$/ \mathrm{s} /$ - [s] [sc' rẽno] sereno & $/ \mathrm{z} /-[\mathrm{z}][$ 'kaza] casa \\
\hline$/ \mathrm{J} /$ - [S] [Ju'visku] chovisco & /3/ - [3] ['zũtas] juntas \\
\hline /1/ - [1] [pi' 'ão] pilão & $/ K /-[K]\left[\bar{g}^{\prime} \mathrm{va} \Lambda \mho\right]$ orvalho \\
\hline$/ \mathrm{r} /$ - [r] [sc'rẽn v] sereno & $/ \mathrm{r} / \mathrm{l}-[\mathrm{r}][$ toriẽsi' aw] torrencial \\
\hline$/ \mathrm{m} /$ - [m] [ma'ñã] manhã & $/ \mathrm{n} /-[\mathrm{n}][\mathrm{n}$ nvi] neve \\
\hline 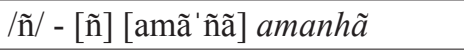 & \\
\hline
\end{tabular}

\subsection{Vocoïdes oraux et nasalisés}

\begin{tabular}{|c|c|}
\hline [a] [kõ' padri] compadre & {$[\varepsilon]\left[\right.$ tẽp $\varepsilon \int^{\prime}$ tadzi] tempestade } \\
\hline [e] [kutu'velv] cotovelo & [i] ['iris] íris \\
\hline [o] [og' 'vaKu] orvalho & [o] ['oKU] olho \\
\hline [u] ['Juva] chuva & [ã] [ãti' õnti] anteontem \\
\hline [ẽ] [sc'rẽnv] sereno & [ĩ] ['iris] íris \\
\hline [õ] ['õntẽy] ontem & [ũ] [ḡidz' mũỹ] redemoinho \\
\hline
\end{tabular}




\subsection{Semivocoïdes oraux}

\begin{tabular}{|l|l|}
\hline [w] ['paw] pau & [y] ['pay] pai \\
\hline
\end{tabular}

\subsection{Tableau des variantes de contoïdes et vocoïdes retrouvées au}

\section{Ceará}

\begin{tabular}{|c|c|}
\hline $\begin{aligned} & {[\mathrm{t}]-\left[{ }^{\prime} \text { tio }\right] \text { tio } } \\
/ \mathrm{t} / & {\left[\mathrm{t} \int\right]-\left[{ }^{\mathrm{t}} \mathrm{f} \mathrm{iw}\right] \text { tio } } \\
& {[\mathrm{t}]-[\mathrm{mi} \text { 'tidu }] \text { metido } }\end{aligned}$ & $\begin{array}{c}{[\mathrm{d}]-[\text { 'dia }] \text { dia }} \\
/ \mathrm{d} /[\mathrm{d} z]-[\text { 'dzia }] \text { dia } \\
{[\mathrm{d}]-[\text { 'dia }] \text { dia }}\end{array}$ \\
\hline 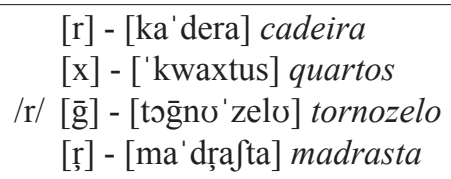 & $\begin{array}{c}/ 1 /[1]-[\text { kutv'velv] cotovelo } \\
{[1]-[\text { ma'linv }] \text { malino }}\end{array}$ \\
\hline $\begin{array}{l}/ \mathrm{n} /[\mathrm{n}]-[\text { ['nadega }] \text { nádega } \\
{[\mathrm{n}]-[\text { mi'nĩno }] \text { menino }}\end{array}$ & $\begin{array}{l}/ \mathrm{a} /[\mathrm{a}]-[\text { 'axko] arco } \\
\quad[\mathrm{a}]-[\mathrm{a} \text { 'maḡga] amarga }\end{array}$ \\
\hline $\begin{array}{l}/ \mathrm{i} /[\mathrm{i}]-[\text { 'tiw }] \text { tio } \\
\quad[\mathrm{i}]-[\mathrm{i} \text { 'dosv }] \text { idoso }\end{array}$ & 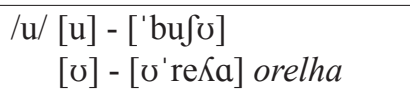 \\
\hline $\begin{array}{l}/ \mathrm{y} /[\mathrm{y}]-\left[\text { 't } \int \mathrm{iyy}\right] \text { tio } \\
{[\tilde{y}]-\left[\text { ['kẽỹt } \int \mathrm{i}\right] \text { quente }}\end{array}$ & $\begin{array}{c}/ \mathrm{w} /[\mathrm{w}]-[\text { ow'vido] ouvido } \\
{[\hat{\mathrm{w}}]-[\text { 'oxfãw] órfão }}\end{array}$ \\
\hline
\end{tabular}

Tous les phonèmes contoïdes sont présents en position initiale, sauf $/ \mathrm{r} /$, /r/ et /ñ / et en position médiane; en position finale, /r/, /s/, /z/, /1/ sont représentés par les variantes $\left[\mathrm{r}, \mathrm{x}, \overline{\mathrm{g}}, \mathrm{r}, \mathrm{s}, \int, \mathrm{z}, 1,1\right]$. Parmi les phénomènes phonétiques observés dans les données cartographiées sont signalés, entre autres :

a) la yodisation du $[\tilde{n}]$ :

redemoinho [ḡidzi' mũño - gidzi' mũy]; calcanhar [kawkã'ña kawkãy'a]; peneirazinha [pẽnera'zĩña - pẽnera'zĩya];

b) la yodisation de $[K]$ :

velha ['veKa - 'veya]; olhos ['oKus - 'oyø]; orvalho [uru'vaKu uru'vay];

c) la réduction du groupe [nd] :

garoando [garo'ãdo - garoâno]; neblinando [neblinãdo - neblinãnช]; serenando [scrẽ'nãdo - scrẽ'nãnu];

d) la vocalisation de [1] en [w] : salgada [sal'gada - saw'gada]; calcanhar [kalkã' ña - kawkã'ña];

e) le rhotacisme de [1] en [r] : ciclone [si'klõni - si'krõni]; neblina [ne'bl ĩna - ni 'br ĩna];

f) l'échange de [n] en [1] : neblineiro [neblĩ' neyro - leblĩ'neru]; 
g) la vocalisation de $[\mathrm{r}]$ en $[\mathrm{w}]$ : arco-íris ['axku 'iris - 'awku 'íris]; órfão ['oxfãw - 'owfãw];

h) la monophtongaison de diphtongues : aguaceiro [agwa'seyru - agwa'serv]; balseiro [baw'seyru baw'seyv]; neblineiro [neblĩ'neyru - neblĩ'nero].

Une analyse des données des enquêtes et des cartes phonétiques laisse apparaître que les contextes socio-éducatifs-culturels favorisent le phénomène de dépalatalisation et de yodisation de $/ K /$ et $/ \tilde{n} /$. Les informateurs analphabètes ou peu alphabétisés, et ceux des groupes d'âges plus élevés, utilisent très souvent la dépalatalisation suivie de yodisation.

\section{Analyse des cartes}

Le volume 2 est divisé en trois grandes parties. La première, très succincte, présente les indications pour la lecture des cartes; la deuxième - il s'agit de l'atlas à proprement parler — rassemble 240 cartes dont 108 cartes lexicales et 132 cartes phonétiques; une troisième partie est consacrée au glossaire divisé à son tour en deux sections, l'une, avec 435 entrées, réunissant les désignations des informateurs scolarisés et l'autre, avec 515 entrées, celles des informateurs analphabètes. Les désignations, en forme orthographique, sont suivies de leur transcription phonétique et, parfois, de deux variantes, entre crochets; sont indiqués aussi les numéros des cartes, lexicales ou phonétiques, relatives aux référents en question et le ou les numéros correspondant aux localités où les désignations ont été relevées. Enfin, une quatrième section présente une bibliographie générale et une cinquième section plus petite avec les références aux sources lexicographiques consultées.

Sur les 306 référents du questionnaire utilisé pour les enquêtes, seuls 46 d'entre eux ont fait l'objet de représentations cartographiques : le critère de sélection de ces derniers a été de présenter une plus grande variation lexicale et phonétique des désignations pour un même référent.

Les cartes prennent en compte, séparément, les réponses des informateurs scolarisés et celles des informateurs analphabètes. Pour toutes les entrées sont ainsi présentées, successivement : a) deux cartes lexicales; b) deux ou trois cartes phonétiques; c) deux nouvelles cartes lexicales ou plus avec des réponses uniques ou autres réponses; d) deux nouvelles cartes phonétiques ou plus avec des réponses uniques ou d'autres réponses. Le nombre de cartes par référent peut ainsi varier de six (p. ex. relampago 'éclair' : cartes 39-44) à 18 (arco iris 'arc-en-ciel'). Les cartes lexicales sont des cartes à symboles : la légende indique les différentes désignations 
ou les différentes variations phonétiques auxquelles elles se rattachent. Sur la carte, les symboles figurent à côté du numéro du point du réseau : leur nombre, traduisant les différentes désignations relevées, peut varier de un à quatre, ce dernier étant cependant assez exceptionnel (on le relève, à titre d'exemple, pour les points 31 et 41 de la carte lexicale 86 , avec les réponses du locuteur analphabète, en réponse à la question arco iris et, pour les points 2, 59 et 69 de la carte 230, avec les réponses des locuteurs scolarisés à la question nàdegas 'fesses'). La présence de désignations multiples, dans un point donné, soulève le problème de la reconnaissance de celle qui peut être considérée comme caractéristique du parler local ou du moins comme la plus fréquemment employée. Dans les cartes qui présentent des désignations uniques ou «autres désignations », ces dernières sont mentionnées seulement dans la légende avec entre parenthèses le numéro du point où elles ont été relevées. Dans la légende des cartes lexicales, les désignations sont présentées en forme orthographique standard; dans les cartes phonétiques, elles figurent en transcription phonétique, mais hors de la légende apparaissent aussi les formes en transcription orthographique. Les cartes sont regroupées en cinq champs sémantiques, à savoir :

- la nature : 43 cartes lexicales et 59 cartes phonétiques (1-102);

- la division du temps : 13 cartes lexicales et 17 cartes phonétiques (103-132);

- la parenté : 17 cartes lexicales et 19 cartes phonétiques (133-168);

- les parties du corps : 35 cartes lexicales et 37 cartes phonétiques (169-240).

L'ordre des cartes suit celui du questionnaire. Dans l'organisation de l'atlas, les cartes lexicales alternent avec des cartes phonétiques correspondant à l'élément lexical analysé.

L'ALECE laisse apparaître une importante variété lexicale pour les référents retenus. Les désignations peuvent être des lexies simples (p. ex. gelu, neve 'neblina') ou des variantes morphologiques de ces dernières, par suffixation entre autres (p. ex. garoa, garoasinha) ou faire partie de syntagmes (p. ex. onda, onda d'agua 'redemoinho'); elles peuvent aussi prendre la forme des lexies complexes, de type descriptif (filhu sem pai sem mãe 'órfão' [orphelin]). On observe aussi que certaines désignations sont en réalité des formes verbales (un gérondif) qui expliquent, par exemple, un phénomène naturel en cours. Ainsi, à côté de neblina (brouillard) on relève (ta) só neblinando (il fait seulement du brouillard; carte 21, p. 48) ou alors les variantes phonétiques réduites liblinando, librinando (ibid., p. 24), neblinando (ibid., p. 18 et 67); pour le référent temporal (orage), à côté de chuva (pluie) ou de chuva grossa (grosse pluie; carte 35), on relève 
des formes comme es(tá) chovendo forte (il est en train de pleuvoir fort; ibid., p. 7) et chovendo grosso (il tombe une grosse pluie; ibid., p. 23). Il peut s'agir enfin de variantes phonétiques comme [nع'blĩna / li'brĩna], ['nadigas / 'nadkgas], [fũ'nil / fũ'niw]. De nombreux référents présentent une grande variété de désignations, dont certaines connaissent de nombreuses variantes, notamment phonétiques. Ainsi, à titre d'exemple, pour le référent nádegas (fesses) on relève 19 types lexicaux; 22 pour les référents tornozelo (cheville) et neblina (brouillard); 37 pour redemoinho (tourbillon, eau qui tourne dans la rivière). Présentant séparément les données relatives à deux catégories d'informateurs, analphabètes et scolarisés, les cartes permettent à la fois de faire l'inventaire des types lexicaux, tous informateurs confondus, mais aussi des types lexicaux employés exclusivement par les locuteurs de chacune des catégories et de ceux qui sont communs aux deux. Prenons comme exemple le référent arco-íris (arc en ciel; voir carte en fin de texte) pour lequel on peut relever, sur les 18 cartes qui lui sont consacrées, les 36 désignations ci-après, répondant à la question «barres colorées que l'on voit dans le ciel après la pluie» : arco, iris, arco-íris, acso-irpe, arco celeste, arco-da-velha, arca-da-aliança, aliançado-céu, cometa, raiolos raios, raio do solo, capelo, carpejo, carrero, torres, torreame, torreano, torre de chuva, torre de capelo, olho de boi, escama de peixe, barralas barras, barra de chuva, barra de vento, barra do dia, (es)tá barreando o tempo, a neve, nevoeiros, as nuve, sinal de nuve, as nuve escura, trovão, relampu, sol, véu. Sur les 36 désignations, 10 sont employées par tous les informateurs, 8 par les seuls informateurs scolarisés et 18 par les informateurs analphabètes qui montrent ainsi une plus grande liberté de création lexicale. Une analyse plus fine de l'ensemble des matériaux relevés permettra sans doute de mieux caractériser les deux catégories d'informateurs, en particulier en référence à la motivation qui est à l'origine des différentes formes lexicales.

\section{Conclusion}

L'Atlas Lingüístico do Ceará constitue une contribution importante à la connaissance de la langue portugaise et des variétés dialectales parlées dans les différentes régions du Nord-Est du Brésil. L'ensemble des matériaux de l'atlas, dont une partie seulement figure dans les deux volumes publiés, par la richesse quantitative et qualitative des données, offre la matière à des futures études approfondies aux niveaux phonético-phonologique, morphosyntaxique, lexical et sémantique et à l'approche motivationnelle dans l'analyse lexicale. Elles rendront possible sans doute une caractérisation précise des parlers de cette région et pourront être utilisées 
par les chercheurs et les enseignants qui travaillent sur la langue portugaise du Brésil. Par ailleurs, l'enregistrement de locuteurs masculins et féminins dans tous les points du réseau devrait permettre la prise en compte d'une troisième variable, diasexuelle.

\section{RÉFÉRENCES BIBLIOGRAPHIQUES}

Aguilera Vanderci de Andrade, 1993, Atlas Lingüístico do Paraná, Londrina, Universidade Estadual de Londrina.

Almeida Edilene, 2009, Atlas Lingüístico da Mata Sul de Pernambuco, mémoire de master, João Pessoa, UFPB.

Aragão Maria do Socorro Silva de \& Menezes Cleusa P. B. de, 1984, Atlas Lingüístico da Paraíba, Brasilia, UFPB /CNPq, Coordenação Editorial, 2 vol.

Bessa José Rogério Fontenele et al., 1982, Questionário do Atlas Lingüístico do Estado do Ceará, Fortaleza, Imprensa da Universidade Federal do Ceará.

Bessa José Rogério Fontenele et al., 2010, Atlas Lingüístico do Estado do Ceará, Fortaleza, UFC, 2 vol.

Brandẽo Sílvia de F., 1991, A Geografia linguística no Brasil, São Paulo, Ática.

CARDoso Suzana Alice Marcelino da Silva, 2005, Atlas Lingüístico de Sergipe II, Salvador, UDUFBA.

CARDoso Suzana Alice Marcelino da Silva et al., 2014, Atlas Lingüístico do Brasil, Londrina, EDUEL, 2 vol.

FERREIRA Carlota et al., 1987, Atlas Lingüístico de Sergipe, Salvador, UFBA, Instituto de Letras, Fundação Estadual de Cultura de Sergipe.

Ferreira Carlota et al., 1994, Diversidade do português do Brasil: estudos de dialetologia rural e outros, Salvador, UFBA.

Lima Fabiana dos Santos, 2009, Atlas Lingüístico do Iguatu - CE, mémoire de master, Fortaleza, UFC.

NASCENTES Antenor, 1958, Bases para a elaboração do Atlas Lingüístico do Brasil, Rio de Janeiro, Casa de Rui Barbosa.

NAscentes Antenor, 1960, O idioma nacional, Rio de Janeiro, Livraria Acadêmica.

Pereira Maria das Neves, 2007, Atlas Geolingüístico do Litoral Potiguar, thèse de doctorat, Rio de Janeiro, UFRJ.

Rossi Nelson, 1963, Atlas Prévio dos Falares Baianos, Rio de Janeiro, INL.

SÁ Edmilson José de, 2013, Atlas Lingüístico de Pernambuco, thèse de doctorat, João Pessoa, UFPB.

Silva Moisés Batista da, 2012, Atlas Lingüístico do Centro-Oeste Potiguar, thèse de doctorat, Fortaleza, UFC.

SiLva Neto Serafim da, 1955, Guia para estudos dialetológicos, Florianópolis, s. éd.

ZáGari Mário Roberto L. et al., 1977, Esboço de um Atlas Lingüístico de Minas Gerais, Rio de Janeiro, Fundação Casa de Rui Barbosa. 


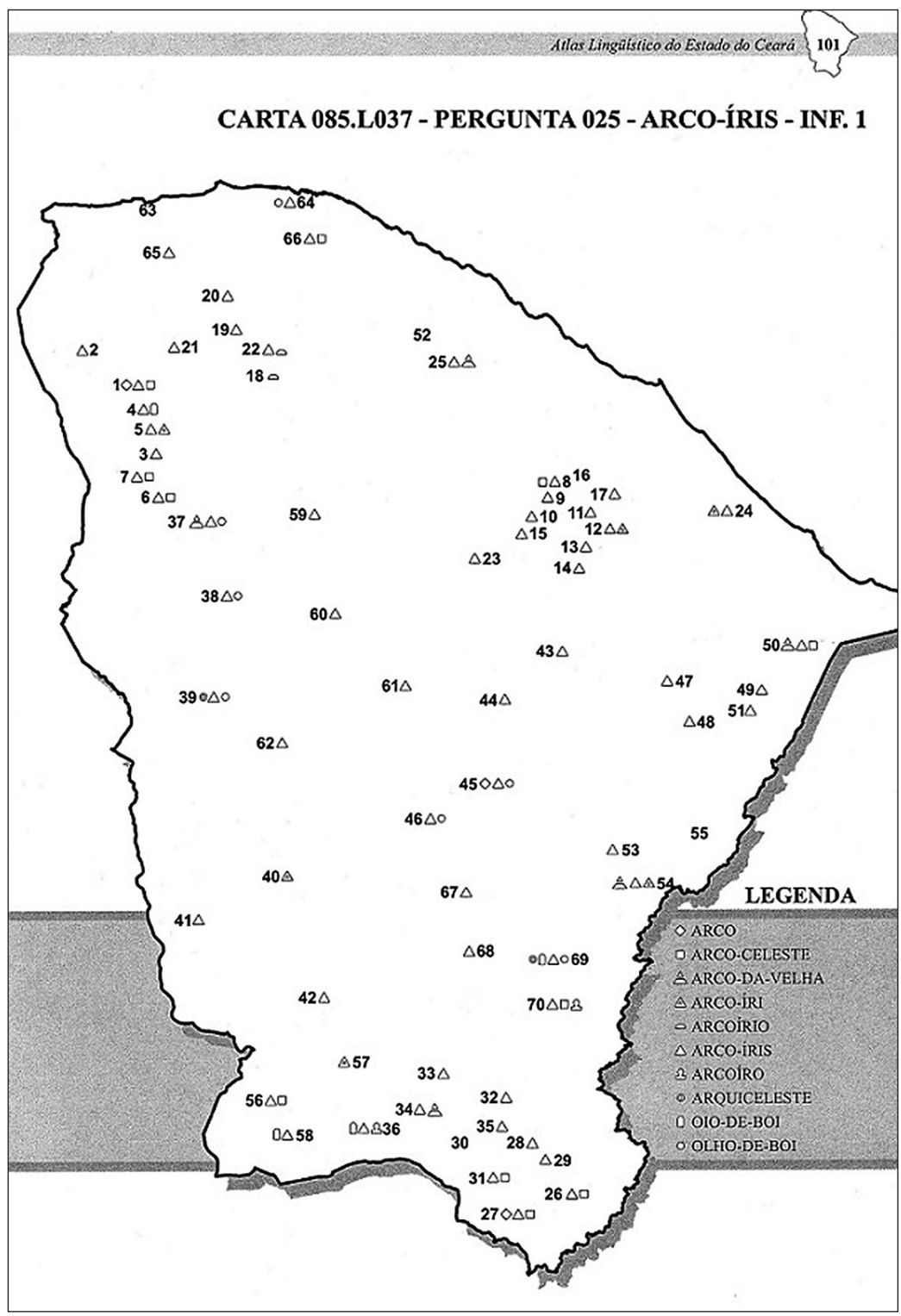

Carte extraite de J.R.F. Bessa et al., 2010, Atlas Lingüístico do Estado do Ceará, vol. 2, Fortaleza, Edições UFC, p. 101. 


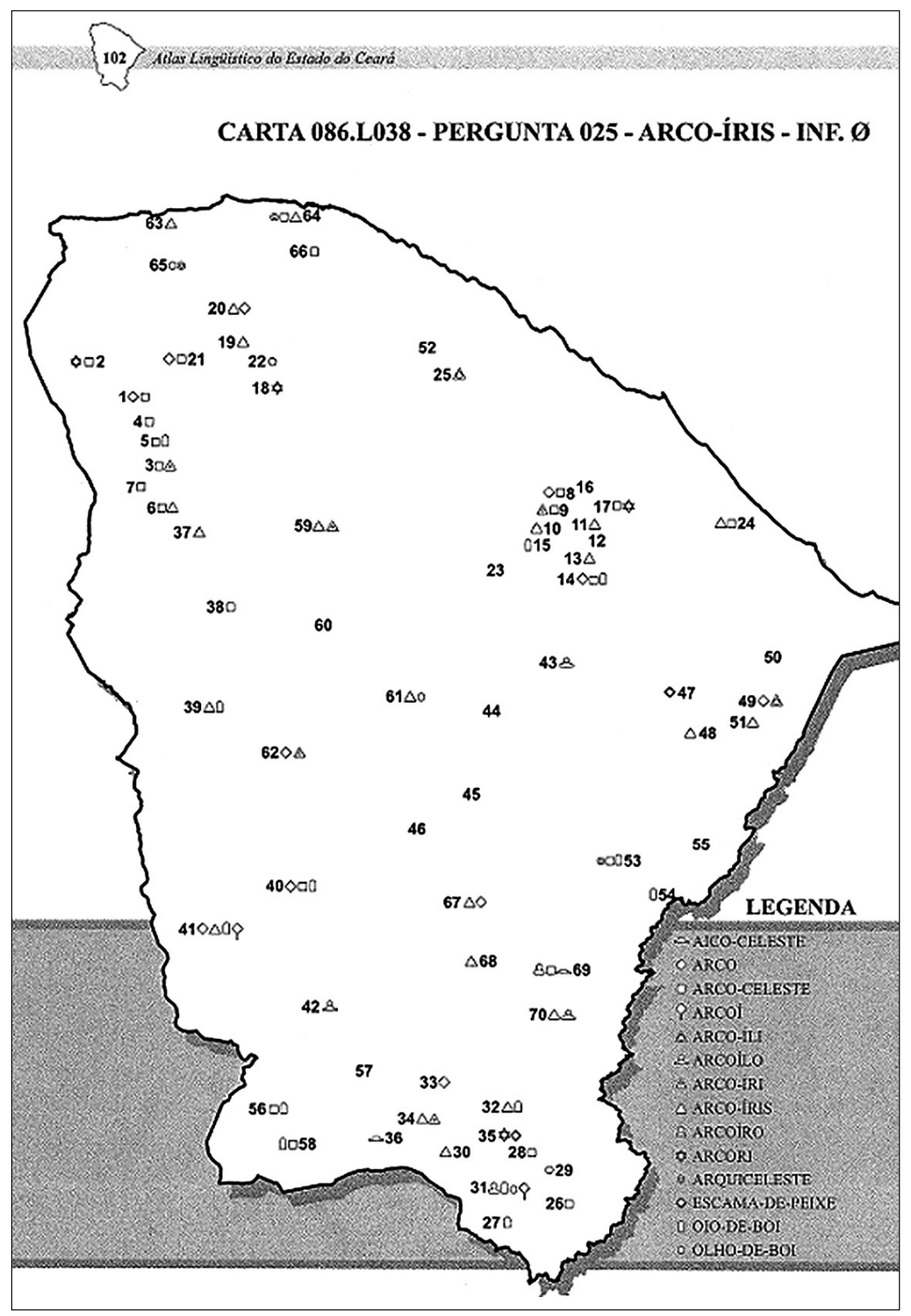

Carte extraite de J.R.F. Bessa et al., 2010, Atlas Lingüístico do Estado do Ceará, vol. 2, Fortaleza, Edições UFC, p. 102. 